\title{
Deep learning augments liver stiffness classification in children
}

\author{
Safwan S. Halabi ${ }^{1}$ (1)
}

Received: 8 October 2020 /Revised: 8 October 2020 / Accepted: 12 November 2020 / Published online: 7 January 2021

(C) Springer-Verlag GmbH Germany, part of Springer Nature 2021

Whenever we find stiffness in the body, our mind should be especially supple. It is never the stiffness in our bodies that limits our practice, it is always the stiffness of our mind.

$$
\text { - Geeta Iyengar }
$$

Let's face it - children are not designed to sit still for long periods of time. We pediatric radiologists, adults, have developed an armamentarium of distractions, sedatives and anesthesia to get patients and their families through medical procedures and diagnostic examinations, including MRI, to minimize the collective angst for everyone involved. We have witnessed incredible innovations in MRI imaging by way of stronger magnets, multichannel and flexible coils, and novel sequences pushing the boundaries of quantitative diagnostics. One of these newer, noninvasive means of quantifying pediatric disease is magnetic resonance (MR) elastography.

There are myriad etiologies of pediatric liver disease including congenital/genetic, acquired and idiopathic. Liver disease can result in scarring of the liver (fibrosis), which increases the stiffness of liver tissue. If liver fibrosis is left unchecked, it can lead to end-stage liver disease or cirrhosis.

Elastography is an imaging technique used to evaluate the mechanical properties of tissue according to the propagation of mechanical waves. MRI or US is coupled with a device that generates mechanical waves, typically shear waves, within the tissues of interest [1]. MR elastography measures the stiffness of liver tissue in children with known or suspected liver disease. Recent studies have shown that normal pediatric liver stiffness corresponds to normal adult liver stiffness [2]. That said, MR elastography and US elastography are not without limitations and pitfalls. Many factors including a child's body habitus [3], food intake prior to the scan, section positioning,

Safwan S. Halabi

safwan.halabi@stanford.edu

1 Department of Radiology, Stanford University, 300 Pasteur Drive, Stanford, CA 94305, USA breathing technique, pulse sequence timing, pulse sequence parameters, and hepatic steatosis can significantly impact image quality and liver stiffness measurement accuracy [1].

Now, what if we can mitigate these variables and stratify liver stiffness predictions by combining known patient clinical data with routine MR imaging techniques? That is exactly what the researchers at Cincinnati Children's Hospital Medical Center aimed to achieve with a study aptly named "DeepLiverNet" [4]. Equipped with a dataset of 273 subjects with known or suspected chronic liver disease that had T2-weighted images and MR elastography stiffness measurements, the authors employed deep learning techniques to predict and classify liver stiffness severity by using patient T2-weighted images of the liver and their clinical information. They used MR elastography stiffness measurements as the gold standard to judge the prediction and classification derived from the routine imaging and clinical data [4].

The results speak for themselves. DeepLiverNet models were assessed with single-channel and multi-channel deep learning methods. Classifying liver stiffness using the T2-weighted imaging data alone achieved an accuracy of $85.2 \%$, with a sensitivity of $66.1 \%$ and specificity of $93.0 \%$. Using the clinical data alone achieved an accuracy of $83.8 \%$, with a sensitivity of $70.9 \%$ and specificity of $89.8 \%$. By combining the T2-weighted imaging data with the clinical data, an accuracy of $88.0 \%$, a sensitivity of $74.3 \%$ and a specificity of $94.6 \%$ were achieved. There are limitations of applying deep learning models derived from a single institution to external datasets in which variables such as varying patient cohorts and disease states and disparate imaging vendors can act as confounding factors that can lead to model overfitting and less accurate predictions. Also, basing the gold standard on imaging quantification instead of pathological diagnosis can bring to question the validity of MR elastography and deep learning that utilize routine imaging data and clinical data to predict liver stiffness. Nevertheless, this is another example of leveraging deep learning in an act of alchemy to convert routine information into gold.

We will undoubtedly continue to see deep learning applications that allow diagnosticians and medical providers to identify patterns and stratify patients without the need for 
time-intensive tests or invasive biopsies. For example, studies have shown that deep learning and machine learning techniques can utilize bone density information extracted from routine CT scans of the body to predict osteoporosis risk $[5,6]$. Also, deep learning was used to predict long-term mortality from a single chest radiograph [7]. The power of deep learning will continue to drive our ability to glean more and more information from fewer and fewer data points and pixels. We need to validate these models in diverse clinical settings to determine whether the models can be generalized. This will ultimately result in shorter scan times, less invasive procedures, and satisfied patients and health care providers.

\section{Compliance with ethical standards}

Conflicts of interest None

\section{References}

1. Guglielmo FF, Venkatesh SK, Mitchell DG (2019) Liver MR elastography technique and image interpretation: pearls and pitfalls. Radiographics 39:1983-2002
2. Trout AT, Anupindi SA, Gee MS et al (2020) Normal liver stiffness measured with MR elastography in children. Radiology. https://doi. org/10.1148/radiol.2020201513

3. Trout AT, Dillman JR, Xanthakos S et al (2016) Prospective assessment of correlation between US acoustic radiation force impulse and MR elastography in a pediatric population: dispersion of US shearwave speed measurement matters. Radiology 281:544-552

4. Li H, He L, Dudley JA et al (2020) DeepLiverNet: a deep transfer learning model for classifying liver stiffness using clinical and T2weighted magnetic resonance imaging data in children and young adults. Pediatr Radiol. https://doi.org/10.1007/s00247-020-04854-3

5. Nam KH, Seo I, Kim DH et al (2019) Machine learning model to predict osteoporotic spine with Hounsfield units on lumbar computed tomography. J Korean Neurosurg Soc 62:442-449

6. Yasaka K, Akai H, Kunimatsu A et al (2020) Prediction of bone mineral density from computed tomography: application of deep learning with a convolutional neural network. Eur Radiol 30:3549 3557

7. Lu MT, Ivanov A, Mayrhofer T et al (2019) Deep learning to assess long-term mortality from chest radiographs. JAMA Netw Open 2: e197416

Publisher's note Springer Nature remains neutral with regard to jurisdictional claims in published maps and institutional affiliations. 